\title{
Treatment of Tumor Lysis Syndrome in Chronic Lymphocytic Leukemia: Protocol for a systematic review and meta-analysis
}

\section{Nancy Kassem}

Hamad Medical Corporation

Ahmed Ghazy

Hamad Medical Corporation

Mohammad A. AbuTineh

Hamad Medical Corporation

Nabil E. Omar ( NOmar4@hamad.qa )

Hamad Medical Corporation https://orcid.org/0000-0001-8291-7987

Abdulqadir J. Nashwan

Hamad Medical Corporation https://orcid.org/0000-0003-4845-4119

\section{Prem Chandra}

Hamad Medical Corporation

\section{Rola S. Ghasoub}

Hamad Medical Corporation

\section{Osama S. AbuTabar}

Cleveland Clinic Abu Dhabi

Mohamed A. Yassin

Hamad Medical Corporation

\section{Protocol}

Keywords: tumor lysis syndrome, Chronic Lymphocytic Leukemia, treatment

Posted Date: August 1st, 2020

DOl: https://doi.org/10.21203/rs.3.rs-41462/v1

License: (c) (i) This work is licensed under a Creative Commons Attribution 4.0 International License. Read Full License 


\section{Abstract}

\section{Background}

Existing evidence on the difference in the incidence of tumor lysis syndrome (TLS) in Chronic Lymphocytic Leukemia (CLL) patients receiving novel therapies versus patients receiving conventional therapies is limited and inconclusive. The aims of this planned systematic review and meta-analysis are therefore (1) assess the TLS incidence reported in clinical trials for the novel or targeted agents comparing to conventional chemotherapeutic agents used to treat patients with CLL (2) to identify the TLS prophylaxis strategies that are utilized in clinical trials of the novel or targeted agents for CLL if it was fully reported or under-reported and (3) to compare the mortality among patients with tumor lysis syndrome in conventional versus novel agents.

\section{Methods}

We will conduct a systematic review and meta-analysis. Several electronic databases will be searched using predefined search terms to identify relevant studies. Eligible studies should report findings on the incidence of TLS in CLL patients. Primary observational studies with cross-sectional or prospective research design, case-control studies, and studies with experimental designs will be included. Study quality will be evaluated by two reviewers using the statistical methodology and categories described in the Cochrane Collaboration Handbook and PRISMA and other applicable guidelines. The meta-analysis will be performed and conducted using applicable standard statistical software like CMA and STATA.

Discussion

This review and meta-analysis will be among the first to systematically explore and integrate the evidence available on the comparison between the incidences of TLS in CLL patients treated with novel agents versus conventional agents. By gathering and summarizing information about the risk of TLS in this patient population, the findings from this review will provide insights for future research directions and more understanding of the difference of TLS incidence between novel treatments and conventional treatment and suggest prophylactic measures for such cases.

Systematic review registration

The protocol has been registered at the International Prospective Register of Systematic Reviews (PROSPERO; registration number: CRD42020166770).

\section{Background}

Chronic lymphocytic leukemia (CLL) is one of the chronic lymphoproliferative disorders (lymphoid neoplasms). It is characterized by a progressive accumulation of functionally incompetent lymphocytes, which are usually monoclonal in origin (1). 
Tumor lysis syndrome is an oncologic emergency that is caused by massive tumor cell lysis with the release of large amounts of potassium, phosphate, and nucleic acids into the systemic circulation (2). Laboratory TLS was defined as any two or more of the following: hyperkalemia, hyperphosphatemia, hyperuricemia, or hypocalcemia, present within three days before or seven days after instituting chemotherapy.

Clinical TLS was defined as laboratory TLS plus one or more of the following that was not directly or probably attributable to a therapeutic agent: increased serum creatinine concentration $(\geq 1.5$ times the ULN), cardiac arrhythmia/sudden death, or a seizure (3).

The incidence and severity of TLS depend on the type of disease and the type of treatment. Most often, it occurs after the initiation of cytotoxic therapy in patients with high-grade lymphomas (particularly the Burkitt's leukemia subtype) and acute lymphoblastic leukemia. However, it can occur spontaneously and with other tumor types that have a high proliferative rate, large tumor burden, or high sensitivity to cytotoxic therapy $(4,5)$.

Multiple effective treatments are available for patients with CLL. However, the risk of TLS associated with treatment hasn't been systemically examined or analyzed. A literature review identified multiple phases I to III clinical trials of Monoclonal antibodies, tyrosine kinase inhibitors, proteasome inhibitors, CAR-T cell therapy, and pro-apoptotic agents like lenalidomide (6-16).

This planned study will systematically review all available literature on the incidence of Tumor Lysis Syndrome (TLS) in patients with Chronic lymphocytic leukemia (CLL) treated with novel versus conventional therapies.

\section{Methods}

The following protocol has been written according to the MOOSE Guidelines for Meta-Analyses and Systematic Reviews of Observational Studies and the PRISMA-P (Preferred Reporting Items for Systematic Reviews and Meta-Analyses) guidelines $(17,18)$. The protocol has been registered at the International Prospective Register of Systematic Reviews (PROSPERO; registration number: CRD42020166770).

\section{Data sources search terms and search strategy}

To achieve the study objectives, searches will be carried out in the following electronic databases: PubMed, Scopus, Web of Science, Web of Conferences, Open Grey. The following search terms will be used in literature review: ("chronic lymphocytic leukemia" OR "well-differentiated lymphocytic lymphoma" OR "small lymphocytic lymphoma" OR "small-cell lymphoma" OR "chronic Lymphoblastic Leukemia" OR "Lymphocytic Lymphoma" OR "Chronic B-Cell Leukemia" OR “Chronic B-Lymphocytic Leukemia" OR "CLL" OR "low-grade lymphoma") AND ("tumor lysis syndrome" OR "tumor lysis syndrome" OR "TLS" 
Reference lists of key full-text and any reports that may be eligible will be reviewed for publications included in the study. The systematic method specifies that all published research constitute the literature search on CLL and TLS. The search strategy is considered adequate to reduce the risk of selection and detection bias. The search results will be exported to Endnote where duplicates are excluded. Included studies will be manually screened to select other relevant studies.

A populated PRISMA-P checklist was used as an aid to authors to clearly, completely, and transparently let reviewers and readers know what authors intend to do (19).

\section{Inclusion and exclusion criteria}

\section{- Types of studies}

Eligible studies should report the empirical incidence of TLS in CLL patients treated with novel and/or conventional therapies. All literature including clinical trials, case reports, case series, and abstracts from 2009 to 2020 in the English language will be included. Data based on conference abstracts and gray literature (e.g., reports, etc.) will also be included. Studies in languages other than English will not be included.

\section{- Participants}

The study population will include adult (18 years or older) patients diagnosed with CLL diagnosis and received treatment. Exclusion criteria include patients aged less than 19 years, known active histological transformation from CLL to an aggressive lymphoma (i.e., Richter's transformation), other diagnoses of active cancer or the presence of other active malignancy or the use of systemic therapy for another malignancy within 3 years; local/regional therapy with curative intent years of treatment is permitted.

\section{Data extraction (selection and coding)}

Two reviewers will independently assess eligibility of all the citations described and extracted data from the original trial reports using a limited data extraction method including the study details for example publication year, authors, study design, clinicalTrials.gov Identifier code, follow-up duration), patient characteristics (inclusion and exclusion criteria and other various related features), sample size and the details of interventions/comparisons, primary and secondary outcome measures, and subgroup/stratified statistical analyses reported included.

Statistical evaluation of both safety and efficacy parameters using various statistical measures such as hazard ratios (HRs), progression-free survival (PFS), and overall survival (OS) between different treatments will be reported.

To minimize any data entry error, all data will be entered in duplicate and cross-checked for accuracy, and disparities would be discussed in a team meeting. Assessment of study quality or strength of study will be carried out as well (high, moderate, low) 


\section{Assessment of methodological quality (risk of bias)}

Study quality will be evaluated by two reviewers using the statistical methodology and categories described in the Cochrane Collaboration Handbook and PRISMA and other applicable guidelines. In case of disagreement, a team meeting/group discussion will be conducted to reach a consensus. Other potential issues will also be considered that includes baseline imbalance and the other potential issues. The cumulative evidence might be affected by bias (e.g., publication bias, selective reporting within studies) will be evaluated. Risk of bias that might affect the cumulative evidence (such as publication bias, selective reporting within studies) will be assessed with plotting the effect by the inverse of its standard error. The symmetry of such 'funnel plots' (using applicable standard statistical software like CMA and STATA etc.) will be assessed both visually, and formally with Egger's test, to see if the effect decreased with increasing sample size.

\section{Meta-analytic approach}

The meta-analysis will be performed and conducted using applicable standard statistical software like CMA and STATA etc. Confidence intervals will be set at $95 \%$. The inter-study heterogeneity will be evaluated with the inconsistency index $\left(I^{2}\right)$. When significant heterogeneity is present $\left(I^{2}>50 \%\right)$, a randomeffects model will be implemented to calculate pooled estimates of specific effect size measures along with the $95 \%$ confidence intervals $(95 \% \mathrm{Cl})$.

Subgroup analysis will be conducted depending on potential factors and covariates that might affect mainly primary outcome measures. Sensitivity analysis will be performed to investigate the effect of individual studies concerning the primary outcome measures of the meta-analysis.

A network meta-analysis will be conducted to compare the treatment outcomes between conventional treatments and several novel targeted agents, and all results will be reported according to the PRISMA extension statement for network meta-analyses.

A network meta-analysis combines direct and indirect estimates of relative treatment effects in one statistical analysis.

A network plot will be produced to represent the data from all trials included in the analysis. The contribution of each direct comparison to the network estimate will be calculated according to the variance of the direct treatment effect and the network structure, later summarized in a contribution plot.

A forest plot of the estimated summary effects, along with Cls for all comparisons, summarizes the relative mean effect or other effect size measure and prediction on each comparison in one plot.

Statistical analysis based on potential subgroups and stratifications (e.g., stage and severity of the disease, age group, gender, clinical significance, and co-morbidity etc.) that might affect primary outcome measures. 


\section{Discussion}

This systematic review and meta-analysis study will estimate the pooled incidence of TLS in patients with CLL with novel and conventional therapies. It will also provide information about the prophylactic measures used. Since this study will use comprehensive and meticulous methods in all the steps of the systematic review and meta-analysis, the information obtained will be completely reliable.

\section{Limitations}

Methodological biases in the primary studies included may cause uncertainty in the results of the present study.

\section{Abbreviations}

TLS

Tumor Lysis Syndrome

CLL

Chronic Lymphocytic Leukemia

CMA

Comprehensive Meta-Analysis

PROSPERO

International Prospective Register of Systematic Reviews

CAR-T

Chimeric antigen receptor $\mathrm{T}$ cell

MOOSE

Meta-analyses of Observational Studies in Epidemiology

PRISMA

Preferred Reporting Items for Systematic Reviews and Meta-Analyses

RCT

Randomized controlled trial

\section{Declarations}

\section{Ethics and dissemination}

Ethical approval is not required for this systematic review and meta-analysis as only a secondary analysis of data already available in scientific databases will be conducted. The results of this review will be submitted for peer-reviewed publication and will be presented at relevant conferences.

\section{Ethics approval and consent to participate}

Not applicable 


\section{Consent for publication}

Not applicable

\section{Availability of data and materials}

Not applicable

\section{Competing interests}

The authors declare that they have no competing interests.

\section{Funding}

This study was not funded.

\section{Authors' contributions}

NK, AG, MAA, NEO, AJN, PC, RG, OSA, MAY: Data Collection, Literature Search, Manuscript Preparation.

All authors read and approved the final manuscript

\section{Acknowledgments}

The publication of this article was funded by the Qatar National Library.

\section{References}

1. Herishanu Y, Katz B-Z, Lipsky A, Wiestner A. Biology of chronic lymphocytic leukemia in different microenvironments: clinical and therapeutic implications. Hematology/Oncology Clinics. 2013;27(2):173-206.

2. Criscuolo M, Fianchi L, Dragonetti G, Pagano L. Tumor lysis syndrome: review of pathogenesis, risk factors and management of a medical emergency. Expert Review of Hematology. 2016;9(2):197208.

3. Cairo MS, Bishop M. Tumour lysis syndrome: new therapeutic strategies and classification. $\mathrm{Br} \mathrm{J}$ Haematol. 2004;127(1):3-11.

4. Cairo MS, Coiffier B, Reiter A, Younes A, Panel TE. Recommendations for the evaluation of risk and prophylaxis of tumour lysis syndrome (TLS) in adults and children with malignant diseases: an expert TLS panel consensus. Br J Haematol. 2010;149(4):578-86.

5. Coiffier B. Acute tumor lysis syndrome-a rare complication in the treatment of solid tumors. Oncology Research Treatment. 2010;33(10):498-9.

6. Advani RH, Buggy JJ, Sharman JP, Smith SM, Boyd TE, Grant B, et al. Bruton tyrosine kinase inhibitor ibrutinib (PCl-32765) has significant activity in patients with relapsed/refractory B-cell malignancies. 
J Clin Oncol. 2013;31(1):88.

7. Barrientos JC, O’Brien S, Brown JR, Kay NE, Reddy NM, Coutre S, et al. Improvement in parameters of hematologic and immunologic function and patient well-being in the phase III RESONATE study of ibrutinib versus ofatumumab in patients with previously treated chronic lymphocytic leukemia/small lymphocytic lymphoma. Clin Lymphoma Myeloma Leuk. 2018;18(12):803-13. e7.

8. Byrd JC, Furman RR, Coutre SE, Flinn IW, Burger JA, Blum KA, et al. Targeting BTK with ibrutinib in relapsed chronic lymphocytic leukemia. N Engl J Med. 2013;369(1):32-42.

9. Chanan-Khan A, Cramer P, Demirkan F, Fraser G, Silva RS, Grosicki S, et al. Ibrutinib combined with bendamustine and rituximab compared with placebo, bendamustine, and rituximab for previously treated chronic lymphocytic leukaemia or small lymphocytic lymphoma (HELIOS): a randomised, double-blind, phase 3 study. The Lancet Oncology. 2016;17(2):200-11.

10. Hallek M, Cheson BD, Catovsky D, Caligaris-Cappio F, Dighiero G, Döhner H, et al. iwCLL guidelines for diagnosis, indications for treatment, response assessment, and supportive management of CLL. Blood. 2018;131(25):2745-60.

11. Herling CD, Cymbalista F, Groß-Ophoff-Müller C, Bahlo J, Robrecht S, Langerbeins P, et al. Early treatment with FCR versus watch and wait in patients with stage Binet A high-risk chronic lymphocytic leukemia (CLL): A randomized phase 3 trial. Leukemia. 2020:1-13.

12. Hoechstetter M, Busch R, Eichhorst B, Bühler A, Winkler D, Eckart M, et al. Early, risk-adapted treatment with fludarabine in Binet stage A chronic lymphocytic leukemia patients: results of the CLL1 trial of the German CLL study group. Leukemia. 2017;31(12):2833-7.

13. O'Brien S, Furman RR, Coutre SE, Sharman JP, Burger JA, Blum KA, et al. Ibrutinib as initial therapy for elderly patients with chronic lymphocytic leukaemia or small lymphocytic lymphoma: an open-label, multicentre, phase 1b/2 trial. The lancet oncology. 2014;15(1):48-58.

14. PIGUET H, MONCONDUIT M, LEBLAY R, GROSBOIS B. JACOMY D. Effects of chlorambucil and therapeutic decision in initial forms of chronic lymphocytic leukemia (stage A): results of a randomized clinical trial on 612 patients. Blood. 1990;75(7):1414-21.

15. trials Coicllam-aotr. CLL Trialists' Collaborative Group. J Natl Cancer Inst. 1999;91:861-8.

16. Woyach JA, Ruppert AS, Heerema NA, Zhao W, Booth AM, Ding W, et al. Ibrutinib regimens versus chemoimmunotherapy in older patients with untreated CLL. New England Journal of Medicine. 2018.

17. Liberati A, Altman DG, Tetzlaff J, Mulrow C, Gøtzsche PC, loannidis JP, et al. The PRISMA statement for reporting systematic reviews and meta-analyses of studies that evaluate health care interventions: explanation and elaboration. J Clin Epidemiol. 2009;62(10):e1-34.

18. Stroup DF, Berlin JA, Morton SC, Olkin I, Williamson GD, Rennie D, et al. Meta-analysis of observational studies in epidemiology: a proposal for reporting. Jama. 2000;283(15):2008-12.

19. Moher D, Shamseer L, Clarke M, Ghersi D, Liberati A, Petticrew M, et al. Preferred reporting items for systematic review and meta-analysis protocols (PRISMA-P) 2015 statement. Rev Esp Nutr Humana y Diet. 2016 Dec 1. 


\section{Supplementary Files}

This is a list of supplementary files associated with this preprint. Click to download.

- PRISMAPchecklist.docx 